\title{
Immune Therapeutics in the Treatment of Advanced Gastric and Esophageal Cancer
}

\author{
ROBIN PARK ${ }^{1}$, STEPHEN WILLIAMSON $^{2}$, ANUP KASI $^{2}$ and ANWAAR SAEED ${ }^{2}$ \\ ${ }^{1}$ Department of Medicine, College of Medicine, Korea University, Seoul, Republic of Korea; \\ ${ }^{2}$ Department of Medicine, Division of Medical Oncology, Kansas University Medical Center, Kansas City, KS, U.S.A.
}

\begin{abstract}
Systemic chemotherapy is the mainstay of therapy in patients with advanced gastric and esophageal cancer, but has multiple drawbacks including lack of durable efficacy and dose limited toxicities. Recent clinical trials data on the efficacy of immune therapy in this patient group have shed light on its potential as an alternative treatment option. Checkpoint inhibitors, specifically the anti-PD-1/PD-L1 inhibitors, seem to be beneficial for a subgroup of patients with advanced gastric or esophageal cancer who have progressed on multiple systemic chemotherapies. As clinical trials results mature, it will become apparent whether checkpoint inhibitors are effective in other treatment settings such as in first-line therapy or adjuvant therapy. Although the toxicity of checkpoint inhibitors is generally unpredictable, they tend to be more manageable and better tolerated than the toxicities of systemic chemotherapy. Furthermore, recent research in molecular subtyping of esophageal and gastric cancer are paving way for better treatment response
\end{abstract}

Abbreviations: MDSCs: Myeloid-derived suppressor cells; Tregs: regulatory $\mathrm{T}$ cells; DCs: dendritic cells; TGF- $\beta$, transforming growth factor beta; IDO: indoleamine-2,3-dioxygenase; IL-10: interleukin 10; IL-2: interleukin 2; IFN- $\gamma$ : Interferon gamma; VEGFA: vascular endothelial growth factor A; VEGFR: vascular endothelial growth factor receptors; PD-1: programmed cell death protein 1; PD-L1: programmed death-ligand 1; CTLA-4: cytotoxic T-lymphocyte associated protein 4; LAG-3: lymphocyte-activation gene 3; TIM-3: T-cell immunoglobulin and mucin-domain containing-3; BTLA: B- and T-lymphocyte attenuator; CSF1R: colony stimulating factor 1 receptor; CAR T cells: chimeric antigen receptor-modified $\mathrm{T}$ cells.

Correspondence to: Anwaar Saeed, MD, Department of Medicine, Division of Medical Oncology, Kansas University Medical Center, GI Oncology Working Group, Kansas City, Kansas City, KS, U.S.A. Tel: +1 913-588-6077, e-mail: asaeed@kumc.edu

Key Words: Immune therapy, gastric cancer, esophageal cancer, check point inhibitor, adoptive cell therapy, cancer vaccine, review. prediction and patient selection for checkpoint inhibitor therapies. Compared to checkpoint inhibitors, other types of immune therapies such as cancer vaccines, and adoptive cell therapies have yet to be proven effective in esophageal and gastric cancer and are further away from clinical use. Immune therapy seems poised to take a firm position as part of the therapeutic armamentarium for advanced gastric and esophageal cancer and future clinical trials will show the extent of its application in different treatment settings in this patient population.

Gastric cancer (GC) and esophageal cancer (EC) are the third and sixth most common cancers in the world and the reported overall survival (OS) for GC and EC is less than 12 months (1-3). Treatment options for advanced GC and EC are limited and responses to such therapies are poor. Immune therapy represents a possible treatment option for advanced EC and GC patients who have exhausted systemic chemotherapy options. Recently published preliminary results from the KEYNOTE-059 trials have led to the first FDA approval of pembrolizumab in advanced esophageal cancer, in the third line setting and the results from the ATTRACTION-2 trial have demonstrated the efficacy of nivolumab in advanced esophageal cancer. The landmark Cancer Genome Atlas (TCGA) study has contributed valuable information in classifying esophageal and gastric cancer into subtypes with distinct immunological phenotypes which may have potential in predicting treatment responses. Based on genomic profiling, 4 distinct subtypes were described: Ebstein-Barr Virus (EBV)associated tumors, microsatellite instability (MSI)-associated tumors, genetically-stable tumors, and tumors with chromosomal instability. The MSI subtype is associated with gene promoter hyper-methylations and exhibit high mutational burden including within the major histocompatibility class I genes. Moreover, EBV-associated tumors are characterized by amplification of genes coding for programmed death-1 (PD-1) and programmed death ligand-1 (PD-L1), suggesting responsiveness to immune therapies targeting PD-1/PD-L1 (4). 
Furthermore, recently published data from the KEYNOTE028 trial suggest that an interferon gamma gene signature may have predictive potential as well. Therefore, immune therapy has gained a foothold in the therapeutic armamentarium for advanced GC and EC and ongoing studies are poised to expand the indication of immune therapy in advanced GC and EC.

Earlier immune therapies such as interleukin-2 (IL-2) and interferon-alpha (IFN- $\alpha$ ) have been in clinical use in melanoma and renal cell cancer since the 1990s. Although such therapies are associated with serious toxicities, they are indicated in only a small subset of patients, and are being used less and less frequently, they have provided the proof-ofconcept that immune therapy can produce durable and meaningful clinical response, thereby paving the way for newer immune therapies (5-7). The newer immune therapies under evaluation for EC and GC are checkpoint inhibitors, cancer vaccines, adoptive cell transfers, and targeted therapies and antibody-drug conjugates. The checkpoint inhibitors currently under evaluation in EC and GC include the PD1/PD-L1 inhibitors such as pembrolizumab, nivolumab, and atezolizumab and the cytotoxic $\mathrm{T}$ lymphocyte associated protein-4 (CTLA-4) inhibitors ipilimumab and tremelimumab. Preliminary results have been reported for the PD-1 and PDL1 inhibitors which demonstrate efficacy and many other clinical trials are under way. Vaccination using various tumorrejection antigens such as tumor oncogenes, cancer-testis antigens, overexpressed proteins, and heat-shock proteins have been evaluated for EC and GC. Adoptive cell transfer therapies (ACTs) using in vitro expanded and activated peripheral blood mononuclear cells (PBMCs), tumorinfiltrating lymphocytes, and chimeric antigen receptor (CAR) $\mathrm{T}$ cells have been evaluated in EC and GC. Both cancer vaccines and ACTs rely on antigen specific recognition of tumor cells as their mechanism of action and as a result, tend to have better adverse effect profiles than the cytokine therapies or checkpoint inhibitors. However, cancer vaccines and adoptive cell transfer therapies (ACTs) have not enjoyed as much success as checkpoint inhibitors in EC and GC and may need to be combined with other immune therapies to produce clinical benefit. The purpose of this review is to outline the different types of immune therapeutics and combination therapies, lay out the existing evidence and ongoing trials evaluating their safety and efficacy, and discuss special considerations that portend to the application of these therapies in the clinical setting.

\section{Checkpoint Inhibitors}

Immune checkpoints exist to maintain the immune system under strict regulation: in order to respond to specific pathogenic microbial insults with appropriate strength, positive checkpoints may dial up while negative checkpoints dial down the immune response (Figure 1). According to the tumor immune-surveillance and immune-editing hypotheses, tumor cells acquire the ability to escape immune surveillance in order to ultimately proliferate and grow out of control (8, 9 ). Tumor cells may achieve this by exploiting negative immune checkpoints such as CTLA-4, which is upregulated on activated $\mathrm{T}$ cells during $\mathrm{T}$ cell priming and leads to $\mathrm{T}$ cell inhibition via interaction with B7.1 and B7.2 (10). For example, tumor cells may recruit regulatory $\mathrm{T}$ cells that constitutively express CTLA-4, thereby limiting the amount of available costimulatory molecules required for $\mathrm{T}$ cell activation (11). Therefore, targeting negative immune checkpoints such as CTLA-4 via monoclonal antibodies may relieve activated $\mathrm{T}$ cells from inhibition thereby restoring antitumor immunity $(12,13)$.

Ipilimumab is a fully human $\operatorname{IgG} 1 \mathrm{~K}$ monoclonal antibody against CTLA-4. A Phase II clinical trial (NCT01585987) evaluated ipilimumab in the second line treatment setting in locally advanced or metastatic gastroesophageal junction cancer. Overall, 114 patients were randomized to receive ipilimumab alone versus best supportive care. No significant difference was found for median overall survival (OS) between the two groups and median progression- free survival (PFS) was lower in the ipilimumab group compared to the best supportive care group (2.9 vs. 4.9 months). Tremelimumab (IgG2 type monoclonal antibody against CTLA-4) was evaluated in a small single center, nonrandomized phase II clinical trial in a second line therapy setting in 18 metastatic GC and EC patients. One patient had a partial response, four had stable disease, and the rest progressive disease. Median OS was 4.8 months and the objective response 5\%. Although the patient who achieved a partial response has shown a remarkably durable response, the overall outcome measures demonstrated that CTLA-4 inhibitor monotherapy has poor efficacy compared to existing second line treatments (14). Therefore, clinical trials are no longer studying monotherapy with anti-CTLA-4 antibodies due to their disappointing results.

PD-1 is a negative immune checkpoint expressed on activated $\mathrm{T}$ cells that binds PD-L1 and PD-L2, resulting in T cell inhibition (15-17). However, PD-1/PD-L1 differs from CTLA-4 in that it functions in the later phases of the adaptive immune response and at the periphery instead of the secondary lymphoid organs $(18,19)$. Furthermore, certain tumors themselves express PD-L1 but not CTLA-4 suggesting that PD-1/PD-L1 is a checkpoint more specific to cancer cells than CTLA-4 (20-23). Such differences may explain the discrepancy in the responsiveness of tumors to therapies targeting CTLA-4 and PD-1/PD-L1. Currently, no clinical trials evaluating anti-PD-1 or anti-PD-L1 drugs in EC and GC are yet complete and only preliminary results are available.

The anti-PD-1 human IgG4 monoclonal antibody nivolumab has been evaluated in the CheckMate-032 study, a 
multi-cohort phase Ib/II trial which enrolled heavily pretreated patients. A total of 59 patients with unknown PD-L1 statuses were given nivolumab alone or nivolumab plus ipilimumab. The patients given nivolumab alone showed an objective response rate (ORR) of $14 \%$. Subsequent PD-L1 testing revealed $38 \%$ of the patients to be PD-L1+. PD-L1+ patients exhibited a superior ORR than their PD-L1 negative counterparts (27\% vs. 12\%). The median duration of response was 7.1 months (24). The efficacy of nivolumab was recently evaluated in the landmark ATTRACTION-2 trial, a multicenter, randomized, double-blind, placebo-controlled phase III trial. The study population consisted of patients with advanced GC or gastroesophageal junction (GEJ) cancer refractory to two or more standard systemic chemotherapies. Four hundred and ninenty-three patients from various centers in Japan, South Korea, and Taiwan were enrolled. The treatment arms were nivolumab $3 \mathrm{mg} / \mathrm{kg}$ versus placebo every 2 weeks. The median OS was 5.26 months $(95 \% \mathrm{CI}=4.60-6.37)$ vs. 4.14 months (3.42-4.86) and the 12-month survival was $26.2 \%$ (20.7-32.0) vs. $10.9 \%$ (6.2-17.0) for nivolumab and placebo respectively. Grade 3-4 TRAEs (treatment related adverse effects) were $10 \%(34 / 330) v s .4 \%(7 / 161)$ and death due to TRAEs 2\% (5/330) vs. 1\% (2/161) respectively (25).

Pembrolizumab is a humanized IgG4 anti-PD-1 monoclonal antibody. The recent KEYNOTE-012 study, a phase Ib trial which enrolled locally advanced or metastatic GC or GEJ cancer patients previously treated with systemic therapy and preselected based on PD-L1 positivity on upfront testing. Thirty-nine patients with GC or GEJ cancer were enrolled. OR was $33 \%$ by central review and $22 \%$ by investigator review. The 6-month progression free survival (PFS) was $24 \%$. The median duration of response was 6 months (26). The KEYNOTE-028 EC cohort included 23 patients. Of these 23 patients, 17 were squamous cell cancers, and 6 were adenocarcinomas and 20 of them had received prior therapies. The OR was $30 \%$ and PFS $30 \%$ at 6 months and $22 \%$ at 12 months. The median duration of response was 10 months (27). The preliminary results of the recent KEYNOTE-059 trial has demonstrated favourable results for pembrolizumab. Cohort 1 enrolled 259 patients who have failed two different previous therapies while cohorts 2 and 3 evaluated 25 and 31 patients, respectively, with newly-diagnosed metastatic or recurrent gastric cancer. The treatment arm consisted of pembrolizumab alone in cohorts 1 and 2 and pembrolizumab plus chemotherapy in cohort 3. Patients were enrolled irrespective of their PD-L1 expression levels except in cohort 3, which only consisted of PD-L1+ patients. The response rate was $16 \%$ in cohort $1,96 \%$ in cohort 2 , and $77 \%$ in cohort 3 . The median overall survival was 5.5 months, 13.8 months, and 20.7 months in cohort 1,2 , and 3 respectively. The favorable response rate seen in cohort 1 , which was expected to be close to 0 , lead to the recent approval of pembrolizumab as a third line treatment for PD-L1+ gastric cancer patients (28-30).

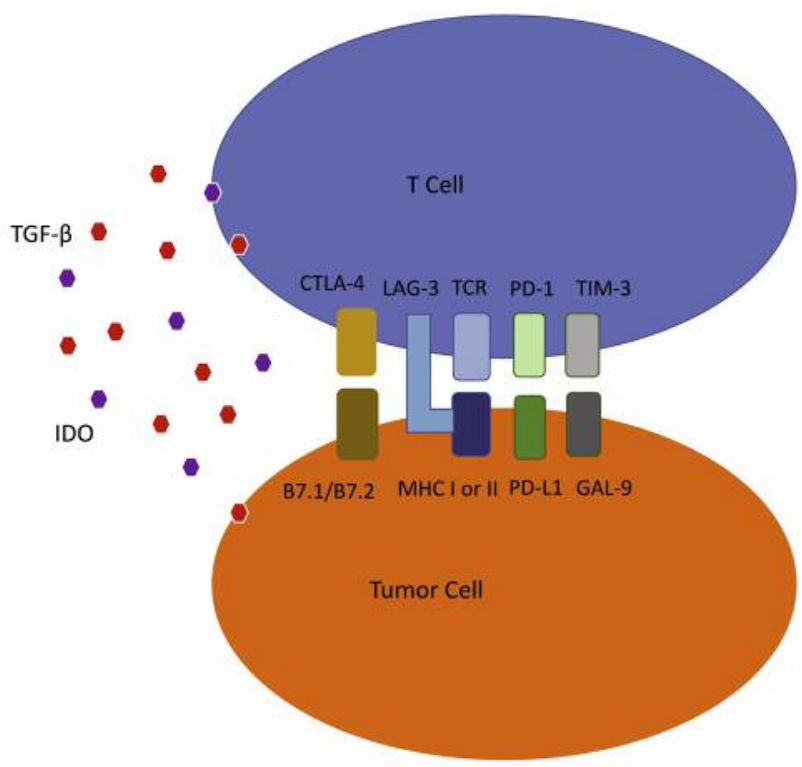

Figure 1. Checkpoint molecules as targets for immune therapies: Immune checkpoint molecules modulate $T$ cell activity by activating or inhibiting intracellular signaling pathways. $P D-L 1$ and $P D-1$ receptor interaction between $T$ cells and tumor cells send inhibitory signals to T cells. CTLA-4 and B7 interaction at the interphase of T cells and APCs prevents activation of $T$ cells. Other checkpoint molecules such as TIM-3 and LAG-3 also modulate $T$ cell intracellular signaling pathways. Soluble immunomodulatory factors such as soluble TGF-beta and IDO may also contribute to $T$ cell inhibition. These multiple mechanisms consequently lead to suppression of anti-tumor immune responses. TCR:, T cell receptor; MHC:, major histocompatibility antigen; TGF- $\beta$ :, transforming growth factor beta; IDO:, indoleamine2,3-dioxygenase; CTLA-4:, cytotoxic T-lymphocyte associated protein 4; LAG-3:, lymphocyte-activation gene 3; PD-1:, programmed cell death protein 1; PD-L1:, programmed death-ligand 1; TIM-3,: T-cell immunoglobulin and mucin-domain containing-3; GAL-9:, galectin-9.

Anti-PD-L1 antibodies have also been evaluated in clinical trials. The IgG1 $\mathrm{K}$ anti-PD-L1 monoclonal antibody durvalumab has demonstrated clinical activity in a Phase I trial and will be evaluated in a dose expansion study for GEJ cancer (31). Avelumab is a human IgG1 lambda monoclonal anti-PD-L1 antibody, which was evaluated in the JAVELIN trial. In this Phase Ib trial, patients with GC or GEJ cancer were given avelumab in either the second-line therapy setting or in a maintenance therapy setting. Seventy-five patients in total received the drug. The overall OR was 9\%. Median PFS in the second line therapy setting was 36 weeks in PD-L1+ patients and 11.6 weeks in PD-L1- patients. In the maintenance group, median PFS for PD-L1+ was 17.6 weeks and 11.6 weeks for PD-L1- (32). Atezolizumab is another fully humanized IgG1 anti-PD-L1 reported to have demonstrated clinical activity in solid tumors, including GC (33). 


\section{Cancer Vaccines}

Cancer vaccines take advantage of antigens associated with tumor cells that may be recognized as foreign by the host adaptive immune system and elicit anti-tumor immune responses $(34,35)$. There are several categories of antigens associated with tumors such as proteins overexpressed in tumor cells, cancer-testis antigens (CTAs), protein products of oncogenes, and heat-shock protein complexes (36). Vaccination strategies have exploited each of these categories of antigens in order to elicit adaptive immune responses and create memory $\mathrm{T}$ and $\mathrm{B}$ cells against tumor cells (37). Human epidermal growth factors receptor-2 (HER-2) is one example of an overexpressed protein which has been exploited for vaccination (38). Dendritic cells pulsed with HER-2 peptides capable of inducing anti-tumor immunity against HER-2 overexpressing GC and $\mathrm{EC}$, were developed in order to be used as vaccines and were evaluated in a phase I trial (38). A total of 9 patients with HER-2/neu-overexpressing tumors and with HLA-A2-positive status were enrolled and vaccinated with dendritic cells pulsed with HER-2 peptide(p369) bi-weekly for 8 weeks. Induction of immune response against HER-2 was evaluated with the interferon-gamma secretion assay, the cytotoxic $\mathrm{T}$ lymphocyte assay, and the peptidespecific delayed-type hypersensitivity response. One patient demonstrated partial response and another patient showed stable disease.

CTAs are proteins expressed only in the testis as well as in certain cancer cells $(39,40)$. Because the testes are a site of immune privilege, the adaptive immune system is not tolerized against these proteins, thereby allowing these proteins to retain their immunogenicity $(39,40)$. NY-ESO-1 is a CTA expressed in both esophageal and gastric tumors. A Phase I trial has evaluated vaccination with NY-ESO-1 in patients with various tumors. Ten patients were enrolled, among which 6 were EC and 1 was a GC patient. All of the patients were vaccinated with NY-ESO-1. The results showed increased antibody responses in 9 out of 10 patients and increased antigen-responsive CD4 and CD8 T cells in all patients. Only 1 patient (EC) overall exhibited stable disease (41). A multi-peptide vaccine consisting of three distinct human-leukocyte antigen (HLA)-A24 binding CTAs, namely TTK protein kinase (TTK), lymphocyte antigen-6-complex locus K (LY6K), and insulin-like growth factor-II (IFG-II), was evaluated in a Phase II clinical trial following promising Phase I trial results (42). The Phase II trial enrolled 60 patients with locally advanced and/or metastatic EC that had failed to respond to therapy. Upon completion of the study, at the point of analysis, the HLA-A typing of the patients were revealed. Results showed that patients harboring the HLA*2402 allele exhibited better PFS than the patients who did not. No difference in OS was seen between the HLA*2402+ versus the HLA*2402- groups, but patients with positive CTL responses to the three peptides displayed better OS than those with negative responses (43).

Heat shock proteins are chaperones that function in binding intracellular proteins. In cancer cells, they can form protein complexes with various deranged intracellular proteins, thereby acting as tumor rejection antigens and inducing CD4+ and CD8+ $\mathrm{T}$ cell responses (44-47). A recent two-arm, open-label, nonrandomized Phase II trial in the adjuvant therapy setting has evaluated vaccination with the heat shock protein gp96 isolated from the tumor cells of 73 GC patients. Patients received either vaccination plus chemotherapy or chemotherapy alone. Disease- free survival (DFS) was higher in the group that received vaccination ( $p=0.045$; hazard ratio: 0.47 ; $95 \%$ confidence interval: 0.23 $0.96)$. 2 -year OS was $81.9 \%$ versus $67.9 \%(p=0.123)$ in the vaccination plus chemotherapy and chemotherapy alone groups respectively (48).

\section{Adoptive Cell Transfer}

Adoptive cell transfer therapies (ACTs) are cell-based therapies that rely on reinfusion of CTLs or natural killer cells (NK cells) to target tumor cells. Accumulating evidence for the use of ACT in hematologic malignancies has led to the recent FDAapproval of ACT in the treatment of a subset of patients in acute lymphoblastic leukemia (49). ACT may use autologous lymphocytes that have been isolated from the blood or from the tumor itself and manipulated in vitro to enhance their activity, or autologous $\mathrm{T}$ cells that have been engineered to express $\mathrm{T}$ cell receptors (TCR) specific for tumor rejection antigens, also called chimeric antigen receptor (CAR) T cells (50). In a Phase I trial, CAR T cells specific for the MAGEA4 antigen have been evaluated in 10 recurrent EC patients. Enrolled patients received the $\mathrm{T}$ cell infusion plus vaccination with the MAGE-A4 antigen. Neither prior lymphodepletion nor IL-2 were given to these patients. Among the 10 patients, 7 displayed progressive disease within 2 months. The remaining 3 patients showed durable responses, but their initial tumor burden was lower compared to the other patients. Thus, the impact of this study's results is unclear. The study's authors have initiated a subsequent study evaluating the treatment modality but with the addition of adjunctive therapies (51).

A study conducted in 2002 evaluated ACT with tumorinfiltrating lymphocytes (TIL) in 44 stage IV GC patients. The patients were divided into chemotherapy-only or ACT pluschemotherapy groups. Lymphocytes were isolated and cultured with an immunological adjuvant prior to injection. The ACT plus chemotherapy group showed a higher overall survival and $50 \%$ survival rates compared to the chemotherapy only group (11.5 vs. 8.3 months). Statistical evaluation demonstrated ACT to be an independent prognostic factor in these patients. Interestingly, the survival benefit was not associated with OR in this trial (52). Another clinical trial 
evaluated peripheral blood mononuclear cells (PBMCs) cultured with cytokines and anti-CD3 in 151 stage III/IV GC patients in the adjuvant setting. The enrolled patients received either chemotherapy alone or ACT plus chemotherapy. Although 5-year OS in these patients was not significantly different, the 5-year DFS was significantly increased in the ACT plus chemotherapy group (28.3\% vs. 10.4\%) (53).

The existing clinical trial data suggest that responses of EC and GC to ACT are poor and the paucity of ongoing trials evaluating ACT reflect these disappointing results. The poor response rate may be explained by induction of immune tolerance in reinfused cells because tumor cells have acquired multiple ways to evade or subvert the immune system. For example, $40 \%$ of esophageal tumor cells express PD-L1 and PD-L2 and infiltration of M2 macrophages into esophageal tumor tissue has been frequently identified. Thus, therapeutic combinations that target multiple mechanisms of tumormediated immune regulation along which include ACT may be needed to overcome the poor responses seen in ACT alone.

\section{Monoclonal Antibodies and Antibody-Drug Conjugates}

HER-2/neu signalling and angiogenesis are two signalling pathways involved in esophageal and gastric cancer that have shown clinical benefit upon therapeutic manipulation. Based on the results of the phase III ToGA trial, trastuzumab was approved for HER-2 positive advanced GEJ cancer. In this trial, 594 patients with previously untreated HER-2 positive GEJ cancer were enrolled. They received either 6 cycles of cisplatin/fluoropyrimdine alone or with trastuzumab. Trastuzumab improved the response rate from 35 to $47 \%$ and prolonged the median OS from 11.1 to 13.8 months. Median OS reached 16.0 months in patients with $\mathrm{IHC}+++$ or IHC2+/FISH+ tumors. No other trastuzumab specific toxicity was observed other than increased frequency of grade III/IV diarrhea (4 vs. 9\%) and asymptomatic reduction in left ventricular ejection fraction (5 vs. 1\%) (54).

The anti-VEGFR-2 monoclonal antibody ramucirumab demonstrated activity in pretreated patients as monotherapy or as a combination therapy with paclitaxel. In the REGARD trial, which enrolled 355 patients who had progressed on firstline chemotherapy, patients were randomized to receive either ramucirumab or placebo. The ramucirumab group showed prolonged OS (5.2 vs. 3.8 months) with a mild increase in toxicity (hypertension $15 \%$ vs. $8 \%$ ) (55). In the RAINBOW trial, 665 patients received either paclitaxel alone or with ramucirumab. Ramucirumab prolonged survival (9.6 vs. 7.4 months) while increasing the frequency of grade III/IV neutropenia (41 vs. 19\%), hypertension (14 vs. 2.5\%) and fatigue (12vs. 5.5\%) (56).

Trastuzumab is approved for HER-2-overexpressing metastatic gastric or gastroesophageal adenocarcinoma in combination with cisplatin and a fluoropyrimidine in patients who have not received prior treatment for metastatic disease. Ramucirumab is approved for use in advanced gastric or gastroesophageal junction adenocarcinoma in combination with paclitaxel and is also approved for use as a single agent in advanced gastric or gastroesophageal junction adenocarcinoma refractory to platinum or fluoropyrimidine based chemotherapy.

Antibody-drug conjugates are novel therapeutic options that exploit the target specificity of monoclonal antibodies to deliver drugs to cancer cells with potent cytotoxicity in order to minimize systemic toxicity. Recently, the safety and activity of trastuzumab deruxtecan (DS-8201), an antibody-drug conjugate consisting of an anti-HER-2/neu monoclonal antibody and a topoisomerase I inhibitor, was evaluated in a multi-center, open-label, dose-escalation phase I trial in Japan, which enrolled and evaluated 23 patients with solid tumors including gastric or GEJ cancer. No dose-limiting toxicities were observed during the study period. The most common serious adverse events were hematotoxicity (grade 3 lymphopenia $(n=3)$, grade 3 neutropenia $(n=2)$, grade 4 anemia $(n=1)$. OR was observed in $43.5 \%(10 / 23)$ of patients and disease control was achieved in $91.3 \%(21 / 23)$ of patients. Median follow up was 6.7 months (57). Further studies are under way to evaluate the safety and efficacy of antibody-drug conjugates in $\mathrm{EC}$ and $\mathrm{GC}$.

\section{Immune-related Adverse Effects}

Because of their immunological mechanism of action, immune therapeutics have an adverse effect profile distinct from those of conventional chemotherapies. Although the most commonly reported symptoms following treatment with checkpoint inhibitor therapy include fatigue, pruritis, arthralgias, and diarrhea, they can lead to more severe complications as a result of their immune-related effects (58). Releasing the adaptive immune system from negative immune checkpoints leads to deranged immune tolerance and may activate preexisting autoreactive $\mathrm{T}$ cells and B cells. Consequently, these therapies can lead to colitis, pneumonitis, dermatitis, and hepatitis as well as endocrinopathies (59). Results, thus far, suggest the adverse effect profiles of checkpoint inhibitors to be superior compared to that of standard of care treatment in EC and GC. For example, nivolumab was associated with a $17 \%$ grade 3 or 4 toxicity rate, while pembrolizumab was associated with a $10 \%$ rate in KEYNOTE 012 and $17 \%$ in the EC cohort of KEYNOTE 028, respectively.

Cancer vaccines are associated with minimal toxicities. Common adverse effects are similar to those associated with vaccination against pathogens such as induration, fatigue, fever, and chills (42). The adverse effect profiles of ACTs are less well defined. In the trial evaluating MAGE-A4 CAR $\mathrm{T}$ cells plus vaccination, none of the 10 patients experienced any adverse effects in the first 14 days after transfer. Mild skin 
reactions associated with vaccination were identified in 4 patients (51). ACT using anti-CD3 and cytokine stimulated PBMCs were associated with various adverse effects. The most common were fever, chills, headache, rash, nausea and vomiting. Adverse effects were entirely manageable by supportive care. Although at face value, these results suggest that ACT is associated with a benign adverse effect profile that ranges from mild to moderate constitutional symptoms in EC and GC, further research and better characterization is needed as serious and fatal toxicities have been reported with the use of ACT in other cancers (60). Nonetheless, the existing data suggest cancer vaccines and ACTs are associated with an adverse effect profile generally milder than other immune therapies.

\section{Immune Therapy Drug Combinations in Treating Gastric and Esophageal Cancer}

Checkpoint inhibitor combinations. Pre-clinical trials and clinical trials have demonstrated improved responses and clinical outcomes with combined immune therapies in advanced melanoma, which has led to the approval of the first immune therapy combination consisting of ipilimumab and nivolumab (61). The rationale for combined immune therapies is based on the notion that combining immune therapeutics with distinct mechanisms of action lead to synergistic enhancement of anti-tumor immunity. For example, CTLA-4 and PD-1/PD-L1 are negative immune checkpoints acting at different spatiotemporal phases of the immune response so that their combined use will lead to a more potent effect on the immune system. Several clinical trials evaluating the efficacy of combination immune therapies are under way for EC and GC. Furthermore, clinical trials are also evaluating the combination of checkpoint inhibitor therapies with cancer vaccines or ACTs (Table I).

Indoleamine-2,3-dioxygenase (IDO) depletes essential amino acids critical for $\mathrm{T}$ cell function and induces differentiation of FoxP3+ regulatory $\mathrm{T}$ cells (Tregs). Expression of IDO by tumor cells and high levels in the tumor microenvironment are associated with poor prognosis. Furthermore, IDO has been postulated as a resistance mechanism against anti-CTLA-4 therapy. Therefore, combining IDO depleting therapies and checkpoint inhibitors may be synergistic in producing tumor responses. There are ongoing trials studying such combination therapies in metastatic melanoma and based on the results of such studies, more trials will be performed in other solid tumors, such as $\mathrm{EC}$ and GC, in the future.

The major hindrance to effective cancer vaccination and ACTs has been eliciting robust and durable immune responses. Checkpoint inhibitors may lower the threshold for initiating immune responses against tumor rejection antigens and result in a more vigorous anti-tumor immune response. Although combination immune therapy increases responses and clinical outcomes, it also leads to an increased frequency and severity of adverse effects. For instance, in clinical trials that evaluated ipilimumab and nivolumab combination therapy in metastatic melanoma $61 \%$ of patients who received the combination therapy reported grade III adverse effects $(61,62)$. Close monitoring and lower thresholds for interventions may be needed in patients undergoing combination immune therapies.

Combining checkpoint inhibitors and conventional chemotherapy. Conventional therapies such as chemotherapy and radiation may have immunomodulatory effects alongside or as a result of their intended effect of cytotoxicity. For example, the large-scale death of cancer cells during chemotherapy and radiation may lead to the release of tumor rejection antigens and danger-associated molecules (63-65). Consequently, the increased availability of antigens and maturation of professional APCs will lead to more effective tumor antigen presentation and thus enhanced anti-tumor immunity. Such immunomodulatory effects suggest that combining chemotherapy and immune therapies may have synergistic effects. Several clinical trials are underway evaluating therapeutic combinations involving conventional therapies and immune therapeutics (Table I).

Combining checkpoint inhibitors and monoclonal antibodies or antibody-drug conjugates. Targeted therapies may also exhibit, in addition to their effects on specific signaling pathways, unintended immuno-modulatory effects. Pre-clinical studies demonstrate that inhibiting the mitogen-activated protein kinase (MAPK) pathway has favourable immunomodulatory effects such as increasing the antigenicity of tumor cells and enhancing the function of T cells (66). The preliminary results of Phase I/II trials evaluating MAPK inhibitor plus anti-CTLA-4 combinations in melanoma suggest favourable tumor responses and tolerability (NCT01767454). Therapies targeting the VEGF pathway have also demonstrated immunomodulatory effects. For example, animal studies show that VEGF pathway inhibition enhances recruitment and infiltration of $\mathrm{T}$ cells into the tumor (67). Consistent with these results, clinical trials have shown that bevacizumab or sunitinib increases tumor-infiltrating lymphocytes, tumor PD-L1 expression, and PD-L1+ tumorinfiltrating lymphocytes, which correlate with improved clinical responses $(66,68)$. Several combination therapies comprised of targeted therapies and immune therapies are under clinical evaluation for EC and GC (Table I).

\section{Predicting Response to Immune Therapy}

Several putative biomarkers for predicting response to checkpoint inhibitor therapy are currently being tested for use in gastric and esophageal cancer. Assessment of PD-L1 
Park et al: Immunotherapy in Advanced GC and EC (Review)

Table I. Ongoing clinical trials evaluating immune therapy in esophageal cancer and gastric cancer.

\begin{tabular}{|c|c|c|c|c|}
\hline \multicolumn{5}{|c|}{ Check Point Inhibitors } \\
\hline Trial & Phase & Therapy & Indication & Status \\
\hline NCT01772004 & $\mathrm{Ib}$ & Avelumab & Various cancers including Gastric and GEJ cancer & Recruiting \\
\hline NCT02625610 & III & Avelumab & Gastric and GEJ cancer & Recruiting \\
\hline NCT01693562 & $\mathrm{I} / \mathrm{II}$ & Durvalumab & Solid tumors & Ongoing but not recruiting \\
\hline NCT02734004 & $\mathrm{I} / \mathrm{II}$ & Durvalumab + olaparib & Various cancers including gastric cancer & Ongoing but not recruiting \\
\hline NCT02340975 & $\mathrm{Ib} / \mathrm{II}$ & Durvalumab + tremelimumab & Gastric and GEJ cancer & Recruiting \\
\hline NCT02267343 & III & Nivolumab & Gastric cancer & Recruiting \\
\hline NCT03006705 & III & Nivolumab & Gastric cancer & Recruiting \\
\hline NCT02903914 & I & Nivolumab + CB-1158 & Various cancers including gastric cancer & Recruiting \\
\hline NCT02864381 & II & Nivolumab + GS-5745 & Gastric cancer & Ongoing but not recruiting \\
\hline NCT02488759 & $\mathrm{I} / \mathrm{II}$ & Nivolumab + ipilimumab & Various cancers including gastric cancer & Recruiting \\
\hline NCT02935634 & II & Nivolumab + ipilimumab & Gastric cancer & Recruiting \\
\hline NCT02872116 & III & Nivolumab + ipilimumab & Gastric and GEJ cancer & Recruiting \\
\hline NCT01928394 & $\mathrm{I} / \mathrm{II}$ & Nivolumab + ipilimumab & Various cancers including gastric cancer & Recruiting \\
\hline NCT02946671 & I & Nivolumab + mogamulizumab & Gastric and esophageal cancer & Recruiting \\
\hline NCT02999295 & $\mathrm{I} / \mathrm{II}$ & Nivolumab + ramucirumab & Gastric and GEJ cancer & Recruiting \\
\hline Keynote 059 & II & Pembrolizumab & Gastric and GEJ cancer & Ongoing but not recruiting \\
\hline Keynote 061 & II & Pembrolizumab & Gastric and GEJ cancer & Enrolling \\
\hline NCT02054806 & I & Pembrolizumab & Solid tumors & Ongoing but not recruiting \\
\hline NCT02559687 & II & Pembrolizumab & Esophageal cancer & Ongoing but not recruiting \\
\hline NCT02268825 & $\mathrm{I} / \mathrm{IIa}$ & Pembrolizumab & Various gastrointestinal Cancers & Ongoing but not recruiting \\
\hline NCT02335411 & II & Pembrolizumab & Gastric and GEJ cancer & Ongoing but not recruiting \\
\hline NCT02494583 & III & Pembrolizumab & Gastric cancer & Ongoing but not recruiting \\
\hline NCT02370498 & III & Pembrolizumab & Gastric and GEJ cancer & Ongoing but not recruiting \\
\hline Keynote 028 & $\mathrm{Ib}$ & Pembrolizumab & Solid tumors & Ongoing but not recruiting \\
\hline Keynote 062 & III & Pembrolizumab + 5-FU & Gastric and GEJ cancer & Recruiting \\
\hline NCT02563548 & I & Pembrolizumab + PEGPH20 & Gastric cancer & Recruiting \\
\hline NCT02443324 & I & Pembrolizumab + ramucirumab & Gastric and GEJ cancer & Ongoing but not recruiting \\
\hline NCT02318901 & $\mathrm{I} / \mathrm{II}$ & Pembrolizumab + trastuzumab & $\begin{array}{l}\text { Various cancers including gastric } \\
\text { and esophageal cancer }\end{array}$ & Ongoing but not recruiting \\
\hline NCT02460224 & $\mathrm{I} / \mathrm{II}$ & PDR001, LAG25 & Various cancers including esophageal cancers & Recruiting \\
\hline \multicolumn{5}{|l|}{ Cancer Vaccines } \\
\hline Trial & Phase & Indication & Therapy & Status \\
\hline NCT00682227 & I & Peptide vaccine & Esophageal cancer & Unknown \\
\hline NCT00995358 & II & Peptide vaccine & Esophageal cancer & Unknown \\
\hline NCT00632333 & I & Peptide vaccine & Esophageal cancer & Unknown \\
\hline NCT01697527 & II & Peptide vaccine + adoptive cell therapy & Esophageal cancer & Recruiting \\
\hline \multicolumn{5}{|c|}{ Adoptive Cell Transfer } \\
\hline Trial & Phase & Indication & Therapy & Status \\
\hline NCT02490735 & II & Non-engineered T cells & Esophageal cancer & Not yet recruiting \\
\hline NCT02490735 & II & Non-engineered $\mathrm{T}$ cells & Esophageal cancer & Not yet recruiting \\
\hline NCT02662348 & I & Non-engineered T cells & Esophageal cancer & Recruiting by invitation \\
\hline NCT02349724 & I & CAR T cells & Various cancers including esophageal cancer & Recruiting \\
\hline NCT02869217 & I & CAR T cells & Various cancers including esophageal cancer & Recruiting \\
\hline NCT01795976 & II & CAR T cells & Esophageal cancer & Ongoing but not recruiting \\
\hline NCT02030561 & $\mathrm{I} / \mathrm{II}$ & $\mathrm{NK}$ cells + trastuzumab & Various cancers including gastric cancer & Recruiting \\
\hline NCT02644863 & II & DC-CIK cells & Esophageal cancer & Recruiting \\
\hline
\end{tabular}

5-FU: 5-Fluorouracil; PEGPH20: pegvorhyaluronidase alfa; GEJ: gastroesophageal junction; CAR T cells: chimeric antigen receptor T cells; NK cells: natural killer cells; DC-CIK cells: dendritic cell-cytokine induced killer cells. 
expression by tumors using commercially approved methods has become standard practice before initiating PD-1/PD-L1 blockade therapy in lung cancer. The recent KEYNOTE-059 trial showed that in the third line setting for gastric cancer, the ORR and CR for PD-L1+ patients treated with pembrolizumab were $22.7 \%$ and $2.7 \%$ respectively, whereas they were $8.6 \%$ and $3.4 \%$ for their PD-L1- counterparts. As it was aforementioned, these findings have led to the approval of pembrolizumab for the third line treatment of PD-L1+ gastric cancer. Ongoing research is evaluating the value of PD-L1 for predicting response to other anti-PD-L1 therapies as well as for predicting response in other treatment settings. The presence of tumor-infiltrating lymphocytes (TILs) in association with PD-L1 expression has been validated in melanoma as a robust biomarker for predicting response to anti-PD-1 therapy, as the presence of both TILs and PD-L1 expression suggest there are tumor-specific CD8+ T cells in the tumor microenvironment under inhibition. Further studies are necessary to evaluate the predictive potential of this biomarker in EC and GC.

The preliminary results of KEYNOTE-059 have demonstrated the potential value of MSI as a biomarker for antiPD-1 therapy. In cohort 1, 174 patients were evaluated for MSI and of them, 7 were deemed MSI-high. Compared to their nonMSI-high counterparts, the MSI-high patients showed better ORR (57.1 vs. 14.3\%), CR (14.3 vs. 2.4\%), and disease control rate $(71.4 v s .22 .2 \%)$. Further research is necessary to validate these findings in a larger number of patients. An 18-gene expression profile score representing $\mathrm{T}$ cell inflammation was also evaluated in KEYNOTE-059 in 144 patients. The results showed that the score was associated with a significantly improved response to pembrolizumab $(p=0.014)(28-30)$.

\section{Further Considerations in Immune Therapy Research}

Currently, biomarkers predicting response to immune therapy for clinical use are remarkably underdeveloped. PD-L1 has proven to be effective in predicting response to anti-PD-1 therapy in non-squamous non-small cell lung cancer patients and is part of routine testing in these patient populations (69). However, the predictability of this biomarker is poor in squamous non-small cell lung cancer patients and highly variable for combination immune therapies (70-74). Several other biomarkers including the presence or absence of tumorinfiltrating lymphocytes (TIL) are under evaluation. A recent study has proposed the use of criteria that rely on PD-L1 expression and TILs to classify tumors on the likelihood of responding to anti-PD-1/PD-L1 therapy (75). The criteria seem to have some value in predicting response in melanoma. However, further studies will be necessary to validate such criteria and other putative biomarkers in other types of cancers including $\mathrm{EC}$ and GC.
The dynamics of response to immune therapy are distinct from that of conventional chemotherapy. Treatment response seems to take a longer time to manifest for immune therapy than for conventional chemotherapy. Furthermore, immune therapy is associated with 'pseudoprogression' which refers to the initial increase in the size of cancer-associated lesions before decreasing in size to reflect treatment response (7678). Because of this reason, measurement of the response via criteria established for conventional chemotherapy such as RECIST 1.1 or WHO criteria are not optimal for immune therapy and thus the immune-related response criteria (irRC) have been established and are already in widespread use for objectively evaluating immune therapeutics (76). Accurate evaluation of tumor responses to immune therapy will depend on fine-tuning of evaluation criteria that take into account the dynamics underlying innate and adaptive immune responses.

The efficacy of combined immune therapies may be enhanced or diminished depending on the scheduling and dosing of the different therapies that are involved. For instance, MAPK signaling inhibitors have demonstrated immunomodulatory effects that are short-lived, such that they fade away after 4 weeks of treatment (79). Administering immune therapy outside of this window may lead to lower efficacy than if it is given concurrently with MAPK inhibitors. On the other hand, immune therapy combinations with chemotherapy or radiation may benefit from sequential regimens with chemotherapy or radiation given first. The rationale is that chemotherapy and radiation may enhance the antigen presentation and consequently $\mathrm{T}$ cell priming, after which the checkpoint inhibitors would be more potent. Thus, the dynamics of immunomodulatory effects is an important consideration when building drug regimens for combined immune therapies (Figure 2).

\section{Conclusion}

Recent advances in immune therapy have led to remarkable improvements in the management of advanced cancer patients who would have been without effective treatments in the past. Numerous clinical trials are now ongoing for the evaluation of immune therapies in EC and GC. These include the checkpoint inhibitors therapies, cancer vaccines, and ACTs. Among checkpoint inhibitors, anti-CTLA-4 therapies have been disappointing as monotherapies, but may be useful when deployed in combination immune therapies. Preliminary results are more promising for anti-PD-1/PD-L1 therapy and suggest that they may be effective treatment options for advanced EC and GC. Immune therapy-related adverse effects are distinct from those of chemotherapies and their management generally relies on modifying the drug dose or administrating immunosuppressive agents. Although 


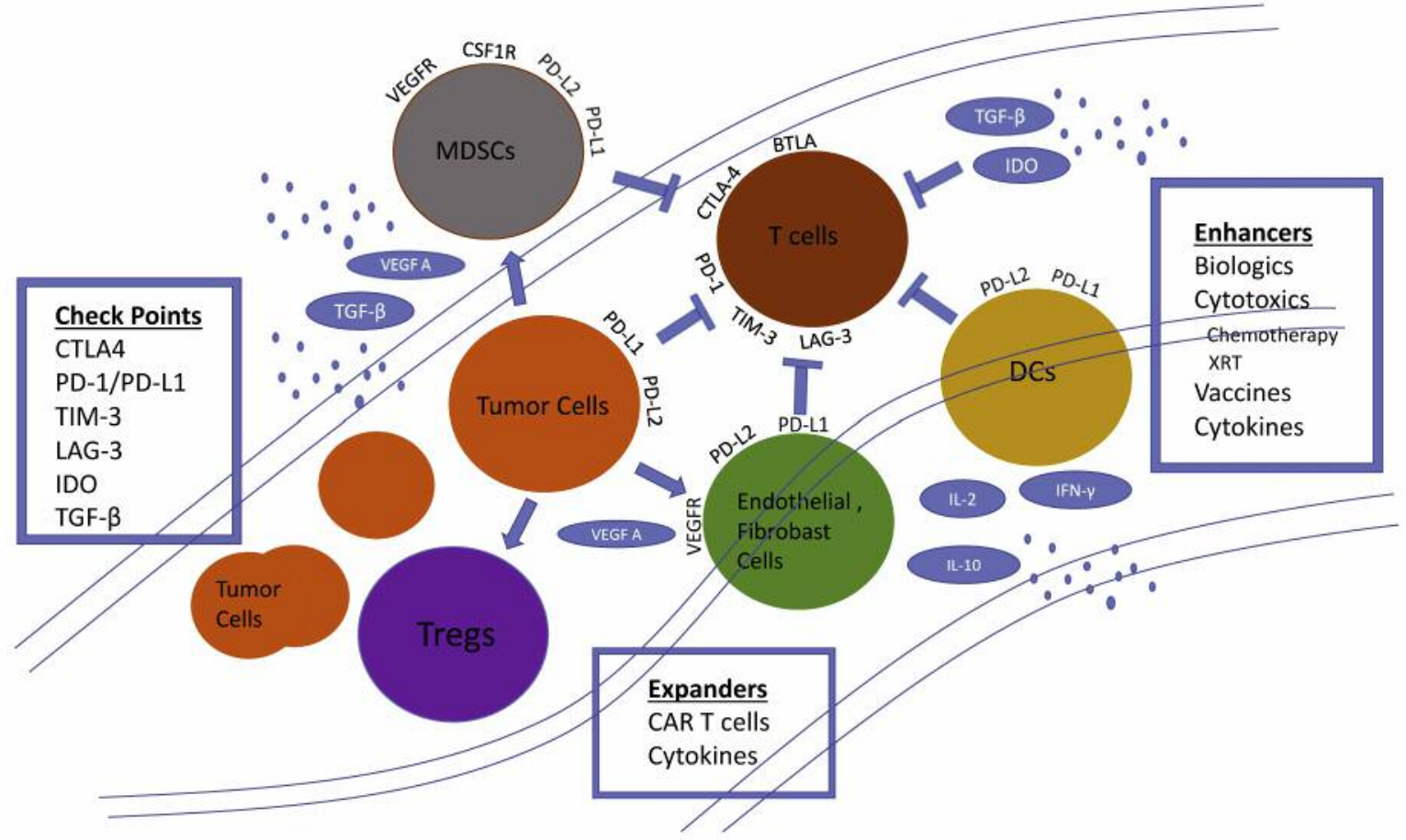

Figure 2. Current landscape of potential modulators of the cancer immunosurveillance system. Several different immune, as well as non-immune cells are involved in modulating the cancer immunosurveillance system. Immunoregulatory cells such as MDSCs and Tregs are recruited to the tumor microenvironment and exert inhibitory effects on tumor-specific cytotoxic T cells. DCs residing in the tumor microenvironment as well as endothelial cells and fibroblasts may also exert T cell inhibitory effects. Such immunomodulatory effects on tumor-specific T cells are mediated by cell surface checkpoint molecules such as CTLA-4 and PD-1/PD-L1 and soluble mediators such as TGF- $\beta$ and IDO. These modulators of the cancer immunosurveillance system are potential targets that may be exploited by various checkpoint inhibitors, immune enhancers, and immune expanders for therapeutic benefit.

cancer vaccines and ACTs are further far from being approved for clinical use, they represent additional immune therapeutic options with different efficacy and safety profiles and warrant further evaluation as combination therapies. Conventional chemotherapy and radiation as well as targeted therapies may also enhance the efficacy of immune therapies. The future direction of immune therapeutic research should address the development of immune therapeutic biomarkers and improveing the evaluation of responses to immune therapy.

\section{Conflicts of Interest}

Anwaar Saeed reports grant funding from AstraZeneca and Exelixis, as well as advisory board fees from Bristol Myers Squibb. Stephen Williamson reports research funding from Merck, Sanofi, and EMD Serono. The remaining Authors report no conflicts of interest.

\section{References}

1 Enzinger PC and Mayer RJ: Esophageal cancer. N Engl J Med 349(23): 2241-2252, 2003.
2 Pisani P, Parkin DM, Bray F and Ferlay J: Erratum: Estimates of the worldwide mortality from 25 cancers in 1990. Int. J. Cancer 83: 18-29, 1999.

3 Wagner AD, Unverzagt S, Grothe W, Kleber G, Grothey A, Haerting $J$ and Fleig WE: Chemotherapy for advanced gastric cancer. Cochrane Database Syst Rev (3): CD004064, 2010.

4 Cancer Genome Atlas Research Network: Comprehensive molecular characterization of gastric adenocarcinoma. Nature 513(7517): 202-209, 2014.

$5 \mathrm{Ma} \mathrm{C}$ and Armstrong AW: Severe adverse events from the treatment of advanced melanoma: a systematic review of severe side effects associated with ipilimumab, vemurafenib, interferon alfa-2b, dacarbazine and interleukin-2. J Dermatolog Treat 25(5): 401-408, 2014.

6 Marabondo S and Kaufman HL: High-dose interleukin-2 (IL-2) for the treatment of melanoma: safety considerations and future directions. Expert Opin Drug Saf 16(12): 1347-1357, 2017.

7 Trinh VA, Zobniw C and Hwu WJ: The efficacy and safety of adjuvant interferon-alfa therapy in the evolving treatment landscape for resected high-risk melanoma. Expert Opin Drug Saf 16(8): 933-940, 2017.

8 Gajewski TF, Meng Y, Blank C, Brown I, Kacha A, Kline J and Harlin H: Immune resistance orchestrated by the tumor microenvironment. Immunol Rev 213: 131-145, 2006. 
9 Dunn GP, Old LJ and Schreiber RD: The immunobiology of cancer immunosurveillance and immunoediting. Immunity 21(2): 137-148, 2004.

10 Wolchok JD and Saenger Y: The mechanism of anti-CTLA-4 activity and the negative regulation of T-cell activation. Oncologist 13: 2-9, 2008.

11 Wang HY, Lee DA, Peng G, Guo Z, Li Y, Kiniwa Y, Shevach EM and Wang RF: Tumor-specific human CD4+ regulatory $T$ cells and their ligands: implications for immunotherapy. Immunity 20(1): 107-118, 2004.

12 Egen JG, Kuhns MS and Allison JP: CTLA-4: new insights into its biological function and use in tumor immunotherapy. Nat Immunol 3(7): 611-618, 2002.

13 Chambers CA, Kuhns MS, Egen JG and Allison JP: CTLA-4mediated inhibition in regulation of $\mathrm{T}$ cell responses: mechanisms and manipulation in tumor immunotherapy. Annu Rev Immunol 19: 565-594, 2001.

14 Ralph C, Elkord E, Burt DJ, O'Dwyer JF, Austin EB, Stern PL, Hawkins RE and Thistlethwaite FC: Modulation of lymphocyte regulation for cancer therapy: a phase II trial of tremelimumab in advanced gastric and esophageal adenocarcinoma. Clin Cancer Res 16(5): 1662-1672, 2010.

15 Topalian SL, Drake CG and Pardoll DM: Targeting the PD-1/B7H1(PD-L1) pathway to activate anti-tumor immunity. Curr Opin Immunol 24(2): 207-212, 2012.

16 Pardoll DM: The blockade of immune checkpoints in cancer immunotherapy. Nat Rev Cancer 12(4): 252-264, 2012.

17 Keir ME, Butte MJ, Freeman GJ and Sharpe AH: PD-1 and its ligands in tolerance and immunity. Annu Rev Immunol 26: 677704, 2008.

18 Ribas A, Chesney JA, Gordon MS, Abernethy AP, Logan TF, Lawson DH, Chmielowksi B, Glaspy JA, Lewis K, Huang B, Wang E, Hsyu PH, Gomez-Navarro J, Gerhardt D, Marshall MA and Gonzalez R: Safety profile and pharmacokinetic analyses of the anti-CTLA4 antibody tremelimumab administered as a one hour infusion. J Transl Med 10: 236, 2012.

19 Shinohara T, Taniwaki M, Ishida Y, Kawaichi M and Honjo T: Structure and chromosomal localization of the human PD-1 gene (PDCD1). Genomics 23(3): 704-706, 1994.

20 Zhou C, Tang J, Sun H, Zheng X, Li Z, Sun T, Li J, Wang S, Zhou X, Sun H, Cheng Z, Zhang $H$ and Ma H: PD-L1 expression as poor prognostic factor in patients with nonsquamous non-small cell lung cancer. Oncotarget $8(35)$ : 5845758468, 2017.

21 Wu Y, Cao D, Qu L, Cao X, Jia Z, Zhao T, Wang Q and Jiang J: PD-1 and PD-L1 co-expression predicts favorable prognosis in gastric cancer. Oncotarget 8(38): 64066-64082, 2017.

22 Kim A, Lee SJ, Kim YK, Park WY, Park DY, Kim JY, Lee CH, Gong G, Huh GY and Choi KU: Programmed death-ligand 1 (PD-L1) expression in tumour cell and tumour infiltrating lymphocytes of HER2-positive breast cancer and its prognostic value. Sci Rep 7(1): 11671, 2017.

23 Chen L, Xiong Y, Li J, Zheng X, Zhou Q, Turner A, Wu C, Lu $\mathrm{B}$ and Jiang J: PD-L1 Expression Promotes Epithelial to Mesenchymal Transition in Human Esophageal Cancer. Cell Physiol Biochem 42(6): 2267-2280, 2017.

24 Janjigian YY, Bendell J, Calvo E, Kim J, Ascierto P, Sharma P, Ott P, Bono P, Jaeger D, Evans TRJ, De Braud F, Chau I, Tschaika M, Harbison C, Lin CS and Le D: CheckMate-032: phase I/II, open-label study of safety and activity of nivolumab (nivo) alone or with ipilimumab (ipi) in advanced and metastatic (A/M) gastric cancer (GC). J Clin Oncol 34(suppl): abstr 4010, 2016.

25 Kang YK, Boku N, Satoh T, Ryu MH, Chao Y, Kato K, Chung HC, Chen JS, Muro K, Kang WK, Yeh KH, Yoshikawa T, Oh SC, Bai LY, Tamura T, Lee KW, Hamamoto Y, Kim JG, Chin K, Oh DY, Minashi K, Cho JY, Tsuda M and Chen LT: Nivolumab in patients with advanced gastric or gastro-oesophageal junction cancer refractory to, or intolerant of, at least two previous chemotherapy regimens (ONO-4538-12, ATTRACTION-2): a randomised, double-blind, placebo-controlled, phase 3 trial. Lancet 390(10111): 2461-2471, 2017.

26 Muro K, Chung HC, Shankaran V, Geva R, Catenacci D, Gupta S, Eder JP, Golan T, Le DT, Burtness B, McRee AJ, Lin CC, Pathiraja K, Lunceford J, Emancipator K, Juco J, Koshiji $\mathrm{M}$ and Bang YJ: Pembrolizumab for patients with PD-L1positive advanced gastric cancer (KEYNOTE-012): a multicentre, open-label, phase $1 \mathrm{~b}$ trial. Lancet Oncol 17(6): 717-726, 2016.

27 Doi T, Piha-Paul SA, Jalal SI, Mai-Dang H, Saraf S, Koshiji M, Csiki I and Bennouna J: Updated results for the advanced esophageal carcinoma cohort of the phase Ib KEYNOTE-028 study of pembrolizumab (MK-3475). Gastrointestinal Cancers Symposium, Poster Session A Board \#F7. J Clin Oncol 34: 7-7, 2016.

28 Bang YJ, Muro K, Fuchs CS, Golan T, Geva R, Hara H, Jalal SI, Borg C, Doi T, Wainberg ZA, Wang J, Koshiji M, Dalal RP and Chung HC: KEYNOTE-059 cohort 2: Safety and efficacy of pembrolizumab (pembro) plus 5-fluorouracil (5-FU) and cisplatin for first-line $(1 \mathrm{~L})$ treatment of advanced gastric cancer. J Clin Oncol 35: 4012, 2017.

29 Catenacci Daniel V, Wainberg Z, Fuchs Charles S, Garrido M, Bang Y-J, Muro K, Savage M, Wang J, Koshiji M, Dalal RP and Kang YK: LBA-009KEYNOTE-059 cohort 3: safety and efficacy of pembrolizumab monotherapy for first-line treatment of patients (pts) with PD-L1-positive advanced gastric/ gastroesophageal (G/GEJ) cancer. Ann Oncol 28: mdx302.008mdx302.008, 2017.

30 Fuchs CS, Doi T, Jang RW-J, Muro K, Satoh T, Machado M, Sun W, Jalal SI, Shah MA, Metges JP, Garrido M, Golan T, Mandala M, Wainberg ZA, Catenacci DVT, Bang Y-J, Wang J, Koshiji M, Dalal RP and Yoon HH: KEYNOTE-059 cohort 1: Efficacy and safety of pembrolizumab (pembro) monotherapy in patients with previously treated advanced gastric cancer. J Clin Oncol 35: 4003, 2017.

31 Zielinski CC: A phase I study of MEDI4736, NNT-PD-L1 antibody in patients with advanced solid tumors. Transl Lung Cancer Res 3(6): 406-407, 2014.

32 Heery CR, O'Sullivan-Coyne G, Madan RA, Cordes L, Rajan A, Rauckhorst M, Lamping E, Oyelakin I, Marte JL, Lepone LM, Donahue RN, Grenga I, Cuillerot JM, Neuteboom B, Heydebreck AV, Chin K, Schlom J and Gulley JL: Avelumab for metastatic or locally advanced previously treated solid tumours (JAVELIN Solid Tumor): a phase 1a, multicohort, doseescalation trial. Lancet Oncol 18(5): 587-598, 2017.

33 Herbst RS, Gordon MS, Fine GD, Sosman JA, Soria JC, Hamid O, Powderly JD, Burris HA, Mokatrin A, Kowanetz M, Leabman M, Anderson M, Chen DS and Stephen Hodi F: A study of MPDL3280A, an engineered PD-L1 antibody in patients with locally advanced or metastatic tumors. J Clin Oncol 31(suppl): abstr 3000, 2013. 
34 Klein G: The strange road to the tumor-specific transplantation antigens (TSTAs). Cancer Immun 1: 6, 2001.

35 Jaffee EM and Pardoll DM: Murine tumor antigens: is it worth the search? Curr Opin Immunol 8(5): 622-627, 1996.

36 Van Der Bruggen P, Zhang Y, Chaux P, Stroobant V, Panichelli C, Schultz ES, Chapiro J, Van Den Eynde BJ, Brasseur F and Boon T: Tumor-specific shared antigenic peptides recognized by human T cells. Immunol Rev 188: 51-64, 2002.

37 Nizard M, Roussel H, Diniz MO, Karaki S, Tran T, Voron T, Dransart E, Sandoval F, Riquet M, Rance B, Marcheteau E, Fabre E, Mandavit M, Terme M, Blanc C, Escudie JB, Gibault L, Barthes FLP, Granier C, Ferreira LCS, Badoual C, Johannes $\mathrm{L}$ and Tartour E: Induction of resident memory $\mathrm{T}$ cells enhances the efficacy of cancer vaccine. Nat Commun 8: 15221, 2017.

38 Kono K, Takahashi A, Sugai H, Fujii H, Choudhury AR, Kiessling R and Matsumoto Y: Dendritic cells pulsed with HER2/neu-derived peptides can induce specific T-cell responses in patients with gastric cancer. Clin Cancer Res 8(11): 3394-3400, 2002.

39 Chaux P, Vantomme V, Stroobant V, Thielemans K, Corthals J, Luiten R, Eggermont AM, Boon T and van der Bruggen P: Identification of MAGE-3 epitopes presented by HLA-DR molecules to CD4(+) T lymphocytes. J Exp Med 189(5): 767778, 1999.

40 Suri A, Saini S, Sinha A, Agarwal S, Verma A, Parashar D, Singh $\mathrm{S}$, Gupta $\mathrm{N}$ and Jagadish N: Cancer testis antigens: A new paradigm for cancer therapy. Oncoimmunology 1(7): 1194-1196, 2012.

41 Kakimi K, Isobe M, Uenaka A, Wada H, Sato E, Doki Y, Nakajima J, Seto Y, Yamatsuji T, Naomoto Y, Shiraishi K, Takigawa N, Kiura K, Tsuji K, Iwatsuki K, Oka M, Pan L, Hoffman EW, Old LJ and Nakayama E: A phase I study of vaccination with NY-ESO-1f peptide mixed with Picibanil OK-432 and Montanide ISA-51 in patients with cancers expressing the NY-ESO-1 antigen. Int J Cancer 129(12): 28362846, 2011.

42 Kono K, Mizukami Y, Daigo Y, Takano A, Masuda K, Yoshida $\mathrm{K}$, Tsunoda T, Kawaguchi Y, Nakamura $\mathrm{Y}$ and Fujii $\mathrm{H}$ : Vaccination with multiple peptides derived from novel cancertestis antigens can induce specific T-cell responses and clinical responses in advanced esophageal cancer. Cancer Sci 100(8): 1502-1509, 2009.

43 Kono K, Iinuma H, Akutsu Y, Tanaka H, Hayashi N, Uchikado Y, Noguchi T, Fujii H, Okinaka K, Fukushima R, Matsubara H, Ohira M, Baba H, Natsugoe S, Kitano S, Takeda K, Yoshida K, Tsunoda T and Nakamura Y: Multicenter, phase II clinical trial of cancer vaccination for advanced esophageal cancer with three peptides derived from novel cancer-testis antigens. J Transl Med 10: 141, 2012.

44 Baker-LePain JC, Sarzotti M and Nicchitta CV: Glucoseregulated protein 94/glycoprotein 96 elicits bystander activation of CD4+ T cell Th1 cytokine production in vivo. J Immunol 172(7): 4195-4203, 2004.

45 Srivastava P: Interaction of heat shock proteins with peptides and antigen presenting cells: chaperoning of the innate and adaptive immune responses. Annu Rev Immunol 20: 395-425, 2002.

46 Binder RJ and Srivastava PK: Peptides chaperoned by heatshock proteins are a necessary and sufficient source of antigen in the cross-priming of CD8+ T cells. Nat Immunol 6(6): 593599, 2005.
47 Wood C, Srivastava P, Bukowski R, Lacombe L, Gorelov AI, Gorelov S, Mulders P, Zielinski H, Hoos A, Teofilovici F, Isakov L, Flanigan R, Figlin R, Gupta R, Escudier B and Group CRS: An adjuvant autologous therapeutic vaccine (HSPPC-96; vitespen) versus observation alone for patients at high risk of recurrence after nephrectomy for renal cell carcinoma: a multicentre, open-label, randomised phase III trial. Lancet 372(9633): 145-154, 2008.

48 Zhang K, Peng Z, Huang X, Qiao Z, Wang X, Wang N, Xi H, Cui J, Gao Y, Huang X, Gao H, Wei B and Chen L: Phase II Trial of Adjuvant Immunotherapy with Autologous Tumorderived Gp96 Vaccination in Patients with Gastric Cancer. J Cancer 8(10): 1826-1832, 2017.

49 Tisagenlecleucel (Kymriah) for ALL. Med Lett Drugs Ther 59(1532): 177-178, 2017.

50 Yeku O, Li X and Brentjens RJ: Adoptive T-Cell therapy for solid tumors. Am Soc Clin Oncol Educ Book 37: 193-204, 2017.

51 Kageyama S, Ikeda H, Miyahara Y, Imai N, Ishihara M, Saito K, Sugino S, Ueda S, Ishikawa T, Kokura S, Naota H, Ohishi K, Shiraishi $\mathrm{T}$, Inoue $\mathrm{N}$, Tanabe $\mathrm{M}$, Kidokoro $\mathrm{T}$, Yoshioka $\mathrm{H}$, Tomura D, Nukaya I, Mineno J, Takesako K, Katayama N and Shiku H: Adoptive transfer of MAGE-A4 T-cell receptor genetransduced lymphocytes in patients with recurrent esophageal cancer. Clin Cancer Res 21(10): 2268-2277, 2015.

52 Kono K, Takahashi A, Ichihara F, Amemiya H, Iizuka H, Fujii $\mathrm{H}$, Sekikawa $\mathrm{T}$ and Matsumoto Y: Prognostic significance of adoptive immunotherapy with tumor-associated lymphocytes in patients with advanced gastric cancer: a randomized trial. Clin Cancer Res 8(6): 1767-1771, 2002.

53 Shi L, Zhou Q, Wu J, Ji M, Li G, Jiang J and Wu C: Efficacy of adjuvant immunotherapy with cytokine-induced killer cells in patients with locally advanced gastric cancer. Cancer Immunol Immunother 61(12): 2251-2259, 2012.

54 Bang YJ, Van Cutsem E, Feyereislova A, Chung HC, Shen L, Sawaki A, Lordick F, Ohtsu A, Omuro Y, Satoh T, Aprile G, Kulikov E, Hill J, Lehle M, Ruschoff J, Kang YK and To GATI: Trastuzumab in combination with chemotherapy versus chemotherapy alone for treatment of HER2-positive advanced gastric or gastro-oesophageal junction cancer (ToGA): a phase 3, open-label, randomised controlled trial. Lancet 376(9742): 687-697, 2010.

55 Fuchs CS, Tomasek J, Yong CJ, Dumitru F, Passalacqua R, Goswami C, Safran H, Dos Santos LV, Aprile G, Ferry DR, Melichar B, Tehfe M, Topuzov E, Zalcberg JR, Chau I, Campbell W, Sivanandan C, Pikiel J, Koshiji M, Hsu Y, Liepa AM, Gao L, Schwartz JD, Tabernero J and Investigators RT: Ramucirumab monotherapy for previously treated advanced gastric or gastro-oesophageal junction adenocarcinoma (REGARD): an international, randomised, multicentre, placebo-controlled, phase 3 trial. Lancet 383(9911): 31-39, 2014.

56 Wilke H, Muro K, Van Cutsem E, Oh SC, Bodoky G, Shimada Y, Hironaka S, Sugimoto N, Lipatov O, Kim TY, Cunningham D, Rougier P, Komatsu Y, Ajani J, Emig M, Carlesi R, Ferry D, Chandrawansa K, Schwartz JD, Ohtsu A and Group RS: Ramucirumab plus paclitaxel versus placebo plus paclitaxel in patients with previously treated advanced gastric or gastrooesophageal junction adenocarcinoma (RAINBOW): a doubleblind, randomised phase 3 trial. Lancet Oncol 15(11): 12241235, 2014. 
57 Doi T, Shitara K, Naito Y, Shimomura A, Fujiwara Y, Yonemori K, Shimizu C, Shimoi T, Kuboki Y, Matsubara N, Kitano A, Jikoh T, Lee C, Fujisaki Y, Ogitani Y, Yver A and Tamura K: Safety, pharmacokinetics, and antitumour activity of trastuzumab deruxtecan (DS-8201), a HER2-targeting antibody-drug conjugate, in patients with advanced breast and gastric or gastrooesophageal tumours: a phase 1 dose-escalation study. Lancet Oncol 18(11): 1512-1522, 2017.

58 Jacquin-Porretaz C, Nardin C, Puzenat E, Roche-Kubler B, Aubin $\mathrm{F}$ and Comite de suivi des effets secondaires des immunotherapies a-c: (Adverse effects of immune checkpoint inhibitors used to treat melanoma and other cancer). Presse Med 46(9): 808-817, 2017.

59 Heinzerling L and Goldinger SM: A review of serious adverse effects under treatment with checkpoint inhibitors. Curr Opin Oncol 29(2): 136-144, 2017.

60 Tey SK: Adoptive T-cell therapy: adverse events and safety switches. Clin Transl Immunology 3(6): e17, 2014.

61 Somasundaram R and Herlyn M: Nivolumab in combination with ipilimumab for the treatment of melanoma. Expert Rev Anticancer Ther 15(10): 1135-1141, 2015.

$62 \mathrm{Kyi} C$ and Postow MA: Immune checkpoint inhibitor combinations in solid tumors: opportunities and challenges. Immunotherapy 8(7): 821-837, 2016

63 Zitvogel L, Galluzzi L, Smyth MJ and Kroemer G: Mechanism of action of conventional and targeted anticancer therapies: reinstating immunosurveillance. Immunity 39(1): 74-88, 2013.

64 Kroemer $G$ and Zitvogel L: Abscopal but desirable: The contribution of immune responses to the efficacy of radiotherapy. Oncoimmunology 1(4): 407-408, 2012.

65 Gameiro SR, Jammeh ML, Wattenberg MM, Tsang KY, Ferrone $\mathrm{S}$ and Hodge JW: Radiation-induced immunogenic modulation of tumor enhances antigen processing and calreticulin exposure, resulting in enhanced T-cell killing. Oncotarget 5(2): 403-416, 2014.

66 Hughes PE, Caenepeel S and Wu LC: Targeted Therapy and Checkpoint Immunotherapy Combinations for the Treatment of Cancer. Trends Immunol 37(7): 462-476, 2016.

67 Motz GT and Coukos G: Deciphering and reversing tumor immune suppression. Immunity 39(1): 61-73, 2013.

68 Guislain A, Gadiot J, Kaiser A, Jordanova ES, Broeks A, Sanders J, van Boven H, de Gruijl TD, Haanen JB, Bex A and Blank CU: Sunitinib pretreatment improves tumor-infiltrating lymphocyte expansion by reduction in intratumoral content of myeloid-derived suppressor cells in human renal cell carcinoma. Cancer Immunol Immunother 64(10): 1241-1250, 2015.

69 Meng X, Huang Z, Teng F, Xing L and Yu J: Predictive biomarkers in PD-1/PD-L1 checkpoint blockade immunotherapy. Cancer Treat Rev 41(10): 868-876, 2015.

70 Borghaei H, Paz-Ares L, Horn L, Spigel DR, Steins M, Ready NE, Chow LQ, Vokes EE, Felip E, Holgado E, Barlesi F, Kohlhaufl M, Arrieta O, Burgio MA, Fayette J, Lena H, Poddubskaya E, Gerber DE, Gettinger SN, Rudin CM, Rizvi N, Crino L, Blumenschein GR Jr., Antonia SJ, Dorange C, Harbison CT, Graf Finckenstein F and Brahmer JR: Nivolumab versus Docetaxel in Advanced Nonsquamous Non-Small-Cell Lung Cancer. N Engl J Med 373(17): 1627-1639, 2015.

71 Larkin J, Chiarion-Sileni V, Gonzalez R, Grob JJ, Cowey CL, Lao CD, Schadendorf D, Dummer R, Smylie M, Rutkowski P, Ferrucci PF, Hill A, Wagstaff J, Carlino MS, Haanen JB, Maio
M, Marquez-Rodas I, McArthur GA, Ascierto PA, Long GV, Callahan MK, Postow MA, Grossmann K, Sznol M, Dreno B, Bastholt L, Yang A, Rollin LM, Horak C, Hodi FS and Wolchok JD: Combined nivolumab and ipilimumab or monotherapy in untreated melanoma. N Engl J Med 373(1): 23-34, 2015.

72 Postow MA, Chesney J, Pavlick AC, Robert C, Grossmann K, McDermott D, Linette GP, Meyer N, Giguere JK, Agarwala SS, Shaheen M, Ernstoff MS, Minor D, Salama AK, Taylor M, Ott PA, Rollin LM, Horak C, Gagnier P, Wolchok JD and Hodi FS: Nivolumab and ipilimumab versus ipilimumab in untreated melanoma. N Engl J Med 372(21): 2006-2017, 2015.

73 Wolchok JD, Kluger H, Callahan MK, Postow MA, Rizvi NA, Lesokhin AM, Segal NH, Ariyan CE, Gordon RA, Reed K, Burke MM, Caldwell A, Kronenberg SA, Agunwamba BU, Zhang X, Lowy I, Inzunza HD, Feely W, Horak CE, Hong Q, Korman AJ, Wigginton JM, Gupta A and Sznol M: Nivolumab plus ipilimumab in advanced melanoma. N Engl J Med 369(2): 122-133, 2013.

74 Brahmer JR, Tykodi SS, Chow LQ, Hwu WJ, Topalian SL, Hwu P, Drake CG, Camacho LH, Kauh J, Odunsi K, Pitot HC, Hamid O, Bhatia S, Martins R, Eaton K, Chen S, Salay TM, Alaparthy S, Grosso JF, Korman AJ, Parker SM, Agrawal S, Goldberg SM, Pardoll DM, Gupta A and Wigginton JM: Safety and activity of anti-PD-L1 antibody in patients with advanced cancer. N Engl J Med 366(26): 2455-2465, 2012.

75 Smyth MJ, Ngiow SF, Ribas A and Teng MW: Combination cancer immunotherapies tailored to the tumour microenvironment. Nat Rev Clin Oncol 13(3): 143-158, 2016.

76 Wolchok JD, Hoos A, O'Day S, Weber JS, Hamid O, Lebbe C, Maio M, Binder M, Bohnsack O, Nichol G, Humphrey R and Hodi FS: Guidelines for the evaluation of immune therapy activity in solid tumors: immune-related response criteria. Clin Cancer Res 15(23): 7412-7420, 2009.

77 Di Giacomo AM, Danielli R, Guidoboni M, Calabro L, Carlucci D, Miracco C, Volterrani L, Mazzei MA, Biagioli M, Altomonte $\mathrm{M}$ and Maio M: Therapeutic efficacy of ipilimumab, an antiCTLA-4 monoclonal antibody, in patients with metastatic melanoma unresponsive to prior systemic treatments: clinical and immunological evidence from three patient cases. Cancer Immunol Immunother 58(8): 1297-1306, 2009.

78 Chiou VL and Burotto M: Pseudoprogression and ImmuneRelated Response in Solid Tumors. J Clin Oncol 33(31): 35413543, 2015.

79 Frederick DT, Piris A, Cogdill AP, Cooper ZA, Lezcano C, Ferrone CR, Mitra D, Boni A, Newton LP, Liu C, Peng W, Sullivan RJ, Lawrence DP, Hodi FS, Overwijk WW, Lizee G, Murphy GF, Hwu P, Flaherty KT, Fisher DE and Wargo JA: BRAF inhibition is associated with enhanced melanoma antigen expression and a more favorable tumor microenvironment in patients with metastatic melanoma. Clin Cancer Res 19(5): 1225-1231, 2013.
Received August 23, 2018 Revised September 11, 2018 Accepted September 12, 2018 